\title{
أنظمة توصيف المنتج البيئي و تأثيرها على الأداء الإقتصادي للمباني
}

\author{
د.م/ عمرو سليمان الجـوهري

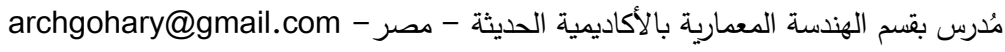

$$
\text { دم/ ديرين عمر خشبة }
$$ \\ Sُدرس بقسم الهندسة المعمارية بالكلية الكندية الدولية CIC - مصر - Shereen_omar@cic-cairo.com
}

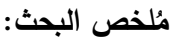

مادة البناء تُعد من العناصر المؤثرة على تطور النتاج المعماري في كل العصور , ومع زيادة تكلفة إنتاج الطاقة في السنوات الأخيرة بسبب الندرة وزيادة الطلب عليها وقلة التطبيقات التي تعتمد علي توظيف الطاقات المتجددة , أدي إلي إرتفاع التكلفة الأولية للمبني بسبب إرتفاع تكلفة الطاقة المُدمجة الأولية , والذي أدي بشكل مباشر إلي عزوف المصممين المعماريين والمستثرين عن التطبيقات

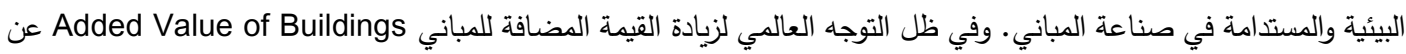

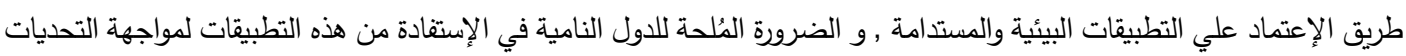

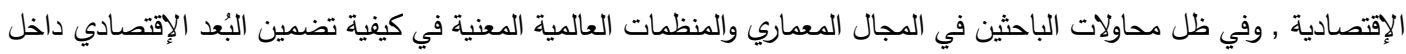
أنظمة تقييم المباني البيئية والمستدامة , كانت فكرة البحث في عمل دراسة لإمكانية تطبيق أنظمة توصيف المنتج البيئي Environmental Product Declarations (EPD) بمثابة بطاقة تعريف تحمل مجموعة من المعلومات عن مواد البناء والمنتجات تُساعد في التعرف علي المادة بشكل يساعد علي لئي

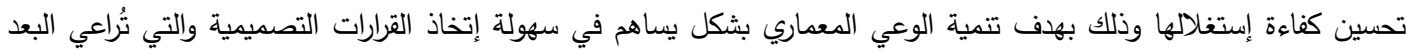

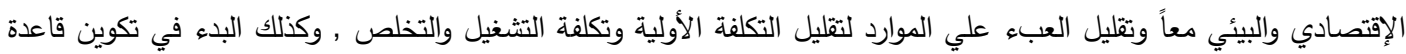
بيانات Data base تحتوي علي توصيف واضح للمواد المحلية المصرية الثائع إستخدامها في صناعة عناصر المبنى المختلفة.

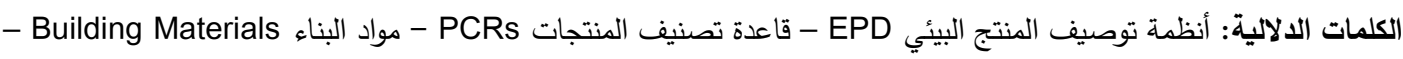
مراحل التكلفة - الطاقة المُدمجة - نظام تقييم المباني البيئية والمستدامة LEED - تقييم دورة حياة المواد المبادئي للمنتجات التي تقدم التحسينات البيئية أو التي تمتلك الثفافيه

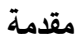

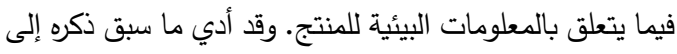
ظهور 439 علامة وشهادة بيئية في 197 دوله ، أي ما يعادل 25 قطاعا صناعيا (1), و من ثم فهذا البحث يتاول إثكالية

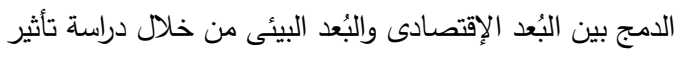

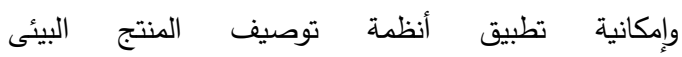
Environmental Product Declarations (EPD) مواد البناء , و مادة البناء هى المكون الأساسي لأى مشروع

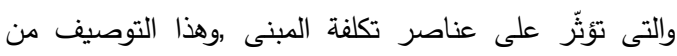
الأساليب التي تتميز بالثفافيه ومُعتَدهد لتوضيح الأثر البيئي

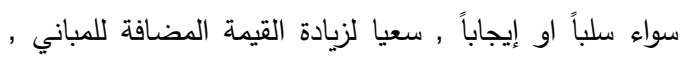
تستهلك صناعة التثييد والبناء كميات كبيرة من المواد والتي مقده تستهلك الكثير من الطاقة في مراحل الإستخراج والتصنيع والتغليف والنقل , ومع تطور العلاقة بين مادة البناء والطاقة في من التئي

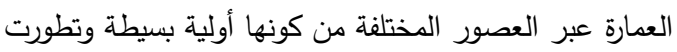

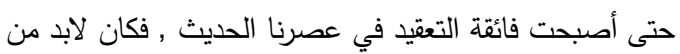

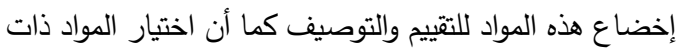
الإستهلاك المرتفع في الطاقة فى مرحلة إنشاء المبنى يؤثر تأثيراً

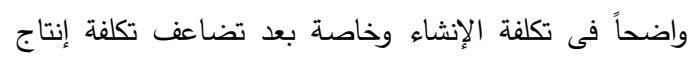

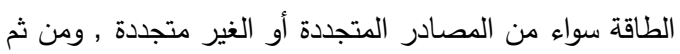
فإن المنظمات الخاصة والمنظمات العامة تعطى الأولوية 
الششروعات ككل , للخروج بمجموعة من النتائج والتوصيات التي تثكل مدخلا للإستفادة من أنظمة توصيف المنتج البيئي

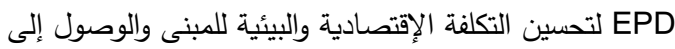
تحقيق أهداف البحث. والثكل رقم (1) يوضح منجية البحث.

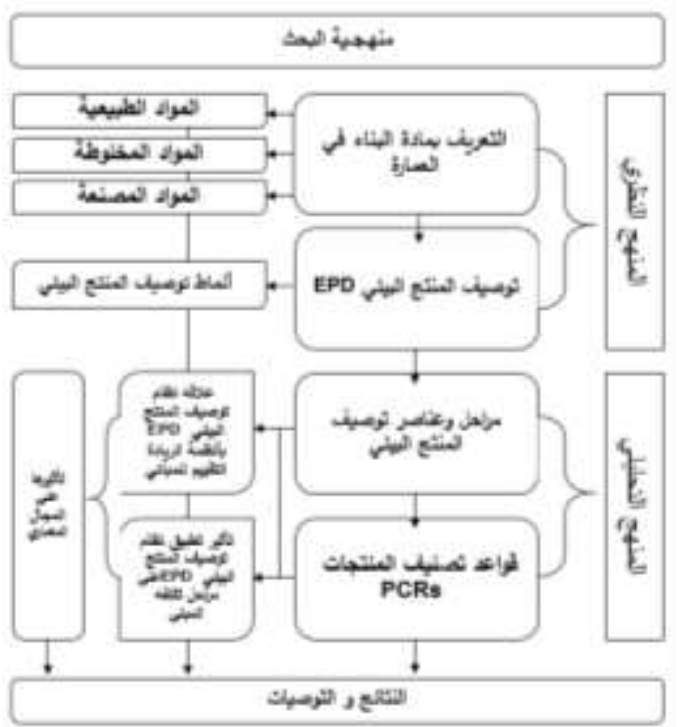

شكل رقم 1 يوضح المنهجية المُتبعه في البحث والتي تعتمد على النى

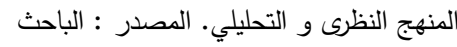

Building Materials in التعريف بمادة البناء في العمارة

\section{Architecture}

هي تلك المواد التي يعتمد عليها المعماري في تجسيد مبانيه, ومع تطور إمكانيات مواد البناء التقليدية وزيادة المعرفة لئية بخصائصها وإمكانياتها الإنثائية والمعمارية بجانب البهاء العوامل

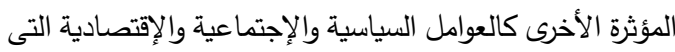

ساعدت على تطور العمارة في العصور المختلفة (2) . وصنفت مواد البناء إلى عدة تصنيفات ولكن من أهمها وأشملها

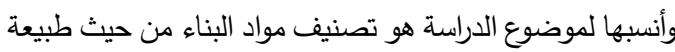
المادة والتي صنفت المادة إلى:- المواد الطبيعية Natural Materials: - المواد العضوية كالأخشاب - مواد التربة)
وتسهيلا لعملية إختيار المنتج والمواد المناسبه مع طبيعه المشروع إلى جانب أنها تؤثر إيجابيا على قيمة المبنى الإقتصاديه.

أهداف البحث وإلمنهجية يستهدف البحث تحقيق مجموعة من الأهداف (الرئيسية

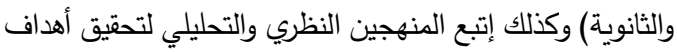

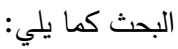
أهداف البحث

يهدف البحث إلي تحويل عملية إنثاء المباني من كونها تقليدية إلي كونها مستدامة عن طريق تحقيق بعض الأهداف الثانوية مثل 1- رفع كفاءة مواد البناء لتحويلها من مجرد مواد تقليدية

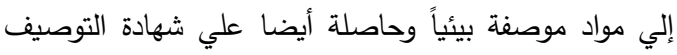

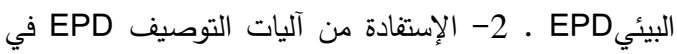

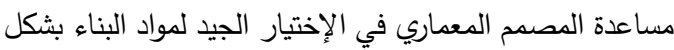
يحسن الأداء الإقتصادي والبيئي للمبني علي مدار دروة حياته.

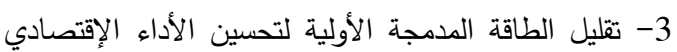

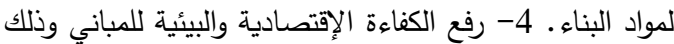
برفع القيمة المضافة. ومن هذا المنطلق يفترض البحث أن تنمية الوعي المعماري

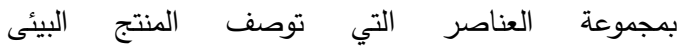
Environmental Product Declarations (EPD) ووضعها في إطار التطبيق العملي يحسن من تكلفة المبنى وخاصة التكلفة الأولية للمبنى , ويحقق إستدامة الموارد البيئية والحد من الخلل في المنظومات البيئية. منهجية البحث إنتهج البحث المنهجين النظري والتحليلي لدراسة مراحل وعناصر إجراء توصيف المنتج البيئي EPD والتركيز علي إرتباط هذه العناصر بالمجال المعماري وعلاقه أنظمة توصيف المنتج الئنج

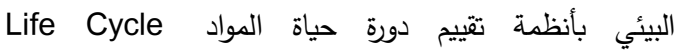
Assessment (LCA)

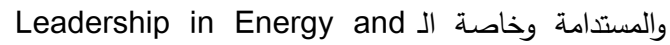
Environmental Design (LEED) العالمية في مؤخرا والأوسع إنتشارا , و تحليل أثره على كالى 
الفعلي كانت فكرة توصيف المنتج البيئي Environmental product declaration (EPD) شكل بطاقه او مُلصق يوضع على المنتج يعمل بمثابة تقرير عن الأداء البيئي للمنتج على مدى دورة حياته، ويتم التقييم بشكل مستقل وحيادي وهي مصممة لتكون نزيهة وليست أداة

تسويقية.

EPD Types أنماط توصيف المنتج البيئي ظهر مفهوم توصيف المنتج البيئي product declaration (EPD) لتوضيح الجوانب البيئية للمنتجات أغلبها فى أطار معايير

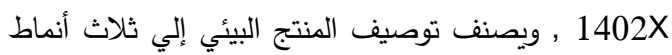

(5) كما هو موضح بالثكل رقم (2).

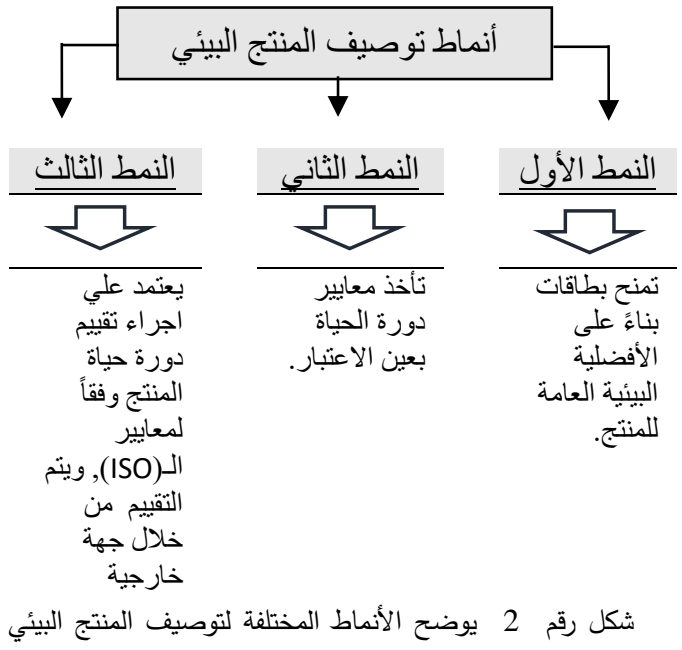

M. Fet et al, "Product المصدر: بتصرف الباحث category rules and environmental product declarations as tools to promote sustainable products": experiences from a case study of furniture production,pp 201, June (2009). من الثكل السابق (2) نجد أن النمط الثالث يعطي معلومات

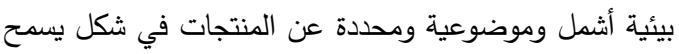
بالمقارنة بين المنتجات التي تحقق وظائف منطابقة لضمان

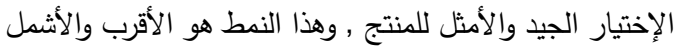

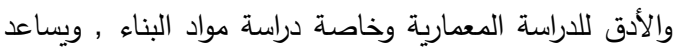

- المواد المخلوطة Mixed Materials: وتثمل (الخرسانة المواد الطينية المخلوطة) - المواد المصنعة Fabricated Materials: (المعادن بأنواعها الحديدية وغير الحديدية - اللدائن - الدائ) (3) (3) (الحريات والسرميكات وتستهلك مادة البناء مجموعة من الطاقات في كل مرحلة من

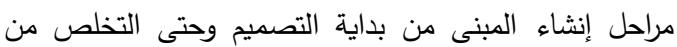
المبنى في نهاية عمره وهي كالأتي: المرحلة الأولى: مرحلة ما قبل البناء : - الطاقة المدمجة Embodied Energy : وهي الاوله هرجله الطاقة المستهلكة في إستخراج وصناعة وتغليف مواد البناء. - الطاقة الرمادية Gray Energy : وهي الطاقة المستهلكة في نقل وتوزيع مواد البناء من المصنع إلى موقع البناء. المرحلة الثانية: مرحلة أثناء البناء: - الطاقة المسببة Induce Energy : وهي الطاقة المستهلكة في عمليات والبناء. - طاقة التثغيل Operating Energy : وهي الطاقة المستهلكة في تشغيل المبنى.

المرحلة الثالثة: مرحلة مابعد البناء (نهاية عمر المبنى): - طاقة التخلص والإحلال Disposal Energy : وهي الطاقة التي تستهلك في هدم والتخلص من المباني في نهاية

عمره (4). توصيف المنتج البيئي (EPD) فى ظل زياده الوعى بالتغير المناخى و قله مصادر الطاقات الغير متجدده و حتمية الأتجاه نحو التصميم البيئى والمستدام

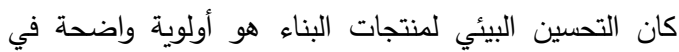
السياسات العالمية, فظهرت الحاجه الى طريقه أو منهج لتقييم

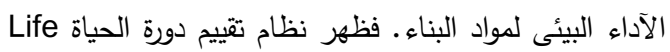
Cycle Assessment (LCA) كطريقه لتتييم تأثير انتاج المواد على البيئه من خلال تتييم دورة

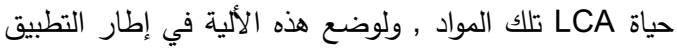


Conduct a life-cycle إجراء تقييم دورة الحياه assessment

إهتمت أكثر من جهة عالمية بإيجاد تعريفات علمية واضحة التقييم دورة الحياة LCA وذلك من خلال إطلاق مبادرة دورة الحياة من قبل مجموعة من الجهات , ولعل من أهمها تعريف

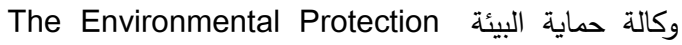
وهو عبارة عن نهج من المه إلى اللحد (EPA) Agency From Cradle to Grave

مراحل دورة حياة المنتجات.

وتعددت متغيرات وأساليب وطرق التقييم بنظام تقييم دورة الحياة في المجال المعماري وذلك حسب طبيعة المنتج المراد توصيفه

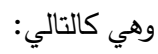

From Cradle to Grave من المهر إلى اللحد

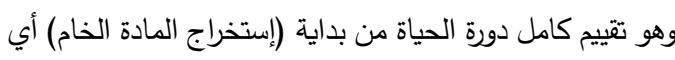
"المها Cradle" مرورا بمرحلة استخدامها (البناء والتشغيل) ووصولا لمرحلة التخلص منها "اللحد Grave" فرول بي نهاية عمر المبنى (8) وهذا الأسلوب أسلوب تقليدي لتقييم دورة حياة مواد

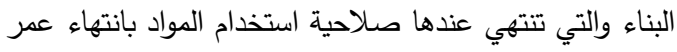
المبني وهدمه وبالتالي تتحول المواد إلي مخلفات صلبة تمثل صلئل

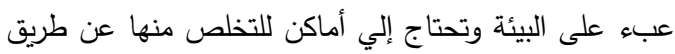

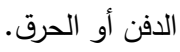

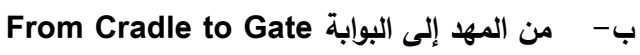
ويقصد "بالبوابة Gate" في هذا الأسلوب ببوابة المصنع فهو

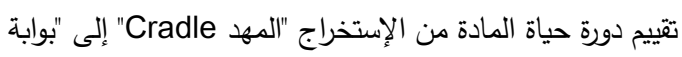

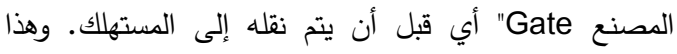

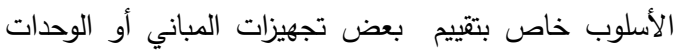

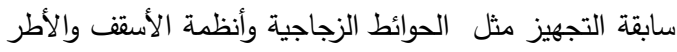
الحاملة وغيرها والتي يتم بعد ذلك إدخالها في إجراء تقييم كامل من المهد الى اللحد في المبنى بأكمله.

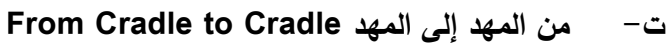

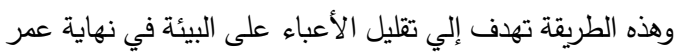

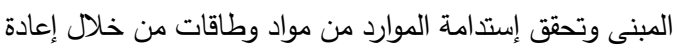

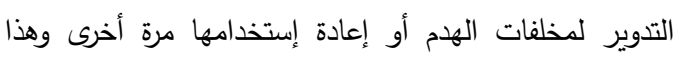

علي تحقيق أهداف البحث , فهو يتيح معرفه الأثر السلبى

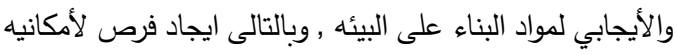
تحسين وتطوير المنتج بيئياً وإقتصاديا وتقليل المخلفات. EPD process أليات إجراء توصيف المنتج البيئى هناك عدة مراحل لتوصيف المنتجات بيئيا وهم: 1- تحديد قواعد

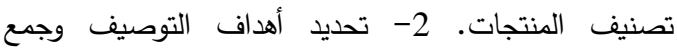
المعلومات 3- إجراء تقييم دورة الحياه. 4- إعداد تقارير توصيف المنتج البيئي (6).

Product Determine تصديد قواعد تصنيف المنتجات Category Rules (PCRs)

وهى مستندات توفر المعلومات والقواعد والمنطلبات والإرشادات

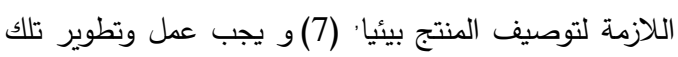

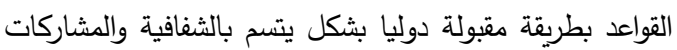

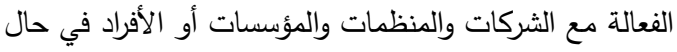
لايهم الخبرة والكفاءة اللازمة لوضع قواعد تصنيف المنتجات وخاصة ما تخص المجال المعماري.

Scoping and تحديد أهداف التوصيف وجمع المعلومات

\section{Data Collection}

تختلف الإستخدامات وتتعدد فيما يخص المواد والمنتجات بثكل

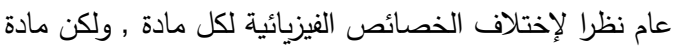
البناء لها طبيعة خاصة تختلف عن أي مادة يمكن أن تستخدم في صناعة أخرى , فرغم أنها تحمل نفس الخصائص الفيزيائية

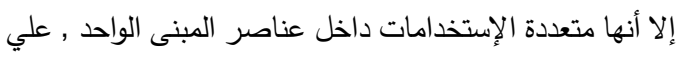

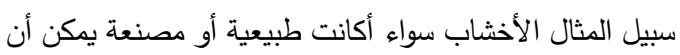
تتعدد إستخداماته داخل أي عنصر من العناصر الستة المكونة لأي مبنى وهي (الموقع - الهيكل الإنشائي - القواطيع الداخلية - الغلاف الخارجي - التثطيات - الفرش والخدمات).

لذلك يجب التعريف الجيد للمنتج وتحديد خصائصة الفزيائية بشكل سليم لكي يتم الإختيار الجيد للمادة المناسبة لعنصر

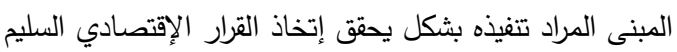

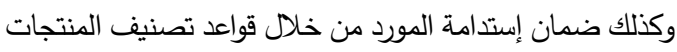
.(PCRs) 
فلابد أن تحمل بطاقة التوصيف معلومات تخص مصادر وأليات إستخراج المواد الخام لأن إختلاف المصادر والأليات يؤثر بشكل

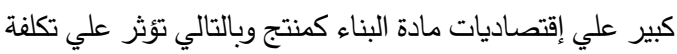
المبني ككل وخاصة التكلفة الأولية.

\section{ب- ب إنتاج المادة الخام Production Process} وتنقسم هذه المرحلة إلي جزئين , الأول خاص بتصنيع المادة

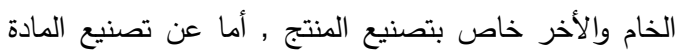

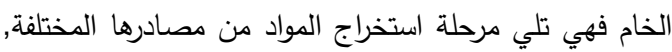

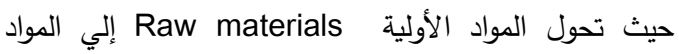
الأساسية Basic Materials وإنتاج المواد ومنتجات البناء المختلفة (9) , وعلي سبيل المثال في مجال التثييد والبناء تحتاج الأحجار إلي عمليات التقطيع والتهذيب والتلميع لتهيأتها للإِتخدام , والمعادن تحتاج إلي عمليات الصهر والتشكيل لإنتاج السبائك. أما عن تصنيع المنتج , فهذا النثاط في مجال التشييد والبناء

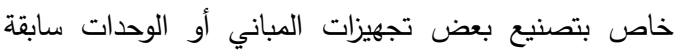
التجهيز مثل الحوائط الزجاجية , والأطر الحاملة , والأجهزة

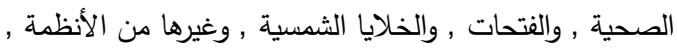

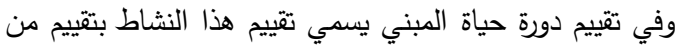
المهد إلي البوابة From Cradle to Gate ويقصد بالبوابة هنا

(بوابة المصنع) الذي يصنع فيه تجهيزات المبنى (10). فلابد أن تحمل بطاقة التوصيف معلومات تخص أليات وأساليب التصنيع لمواد وعناصر المبني المختلفة لأن إختلاف تكنولوجيا التصنيع تؤثر بشكل كبير علي إقتصاديات مادة البناء كمنتج وبالتالي تؤثر علي تكلفة المبني ككل وخاصة التكلفة الأولية.

ت ت -نقل وتعبئة وتغليف المنتجات Product Handling تعتبر من أهم العناصر التي تؤثر علي إقتصاديات المنتج ,

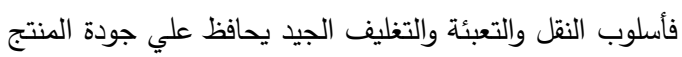
ويقلل الهالك , وتسمى الطاقة التي تستهلك في عمليات التوزيع والنقل كما ذكرنا في تعريف مادة البناء في العمارة بالطاقة الرمادية Gray Energy.
From الأسلوب مثل الأسلوب الأول "من المهد إلى اللحد Cradle to Grave دورة حياته تكون بداية عملية إعادة التدوير . ومن عملية إعادة التدوير تتشأ منتجات جديدة مطابقة أو مختلفة تثكل بداية دورة حياة جديدة ليحقق مفهوم الدائرة المغلقة From Cradle أو من المهذ إلى المهيد Closed Cycle , والتي تساهم بشكل كبير في استدامة مواد البناء , to Cradle والحفاظ علي مصادرها (10). فلابد من أن تحمل بطاقة التوصيف معلومات تخص تقييم دورة

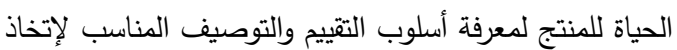
القرارات الإقتصادية والبيئية السليمة.

Prepare report for إعداد تقارير توصيف المنتج البيئي EPD

تحتوى بطاقة التوصيف البيئى للمنتج على مجموعة من المعلومات الأساسيه والمتغيرة بسبب تعدد إستخدامات المواد والمنتجات بشكل عام وخاصة في المجال المعماري ولإختلاف ظروف كل مادة من المواد وهذه المعلومات مرتبطة بدورة حياة

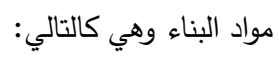

أ- إستخراج ومصادر المواد الخام Extraction of Raw

\section{Materials}

إستخراج المادة الخام من مصادرها هي عملية البحث عن المصادر المناسبة للمواد الخام بشكل يحقق كميات كبيرة من الخام بأقل استخدام للمعدات وبأقل إستهلاك للطاقة لتحسين لتحنين إقتصاديات المادة المراد إستخراجها ,ففي المجال المعماري وقطاع التشييد والبناء فهي صناعة تعمل على استغلال المحاجر

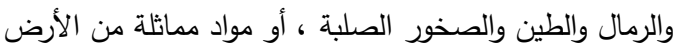

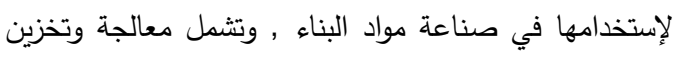

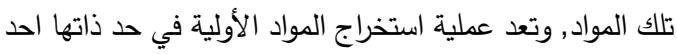
أهم مصادر تدمير البيئة الطبيعية , سواء من عملية التعدين كما

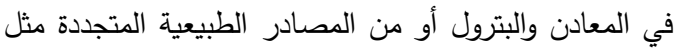
الأخشاب , و تتطلب عملية الإستخراج كميات كبيرة من الطاقة لتثغيل المعدات. 


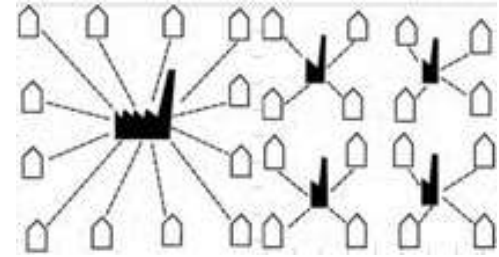

شكل رقم 3 يوضح الفرق بين المركزية واللامركزية في تصنيع

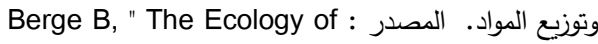
Building Materials",P19, (2009).

لذلك يجب أن تحمل بطاقة التوصيف معلومات تخص أسلوب نقل المنتج والوسيلة المستخدمة وطريقة التعبئة والتغليف بكل شفافية لأن ذلك التوصيف سوف يشجع علي الإعتماد علي المادة المحلية وإتخاذ القرارات التصميمية السليمة التي تقلل من

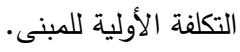

\section{Use Age ث-العمر الإفتراضي للمنتج} لكل منتج من المنتجات أو أي مادة من المواد لها بعمر إفتراضي طبقا لخصائها الفيزيائية وأسلوب تركيبها وإستخدامها , فطبيعة المباني كمنتجات أنها يمكن أن تبقي لعشرات بل مئات السنين, وهذا ما وضعنا في أشكالية إقتصادية وبيئية بسبب إفتقار الوعي المعماري لإدراك العلاقة بين العمر الإفتراضي والعمر الإنتفاعي لأي مبني , فأثبتت الدراسات التحليلية علي عينات عشوائية لمجموعة من المباني أن العمر الإنتفاعي يمثل ثلث العمر الإفتراضي فيتراوح العمر الإفتراضي لأي مبنى حوالي 100 عام في حين أن الدراسات أثبتت أن العمر الإنتفاعي للمبنى في الإسي المتوسط حوالي 30 عام , فيتعرض هذا المبنى للهدم لإعادة إستغلاله مرة أخري في وظائف جديدة. فكان لابد من أن تحمل بطاقة التوصيف معلومات تخص العمر الإفتراضي للمواد والمنتجات لكي يتم الإختيار الجيد للمواد وإتخاد القرار الإقتصادي والبيئي السليم ومساعدة المعماري في صناعة تصاميم معمارية تحترم العلاقة بين العمر الإفتراضي والعمر الإنتفاعي بشكل يؤثر بشكل إيجابي علي قراره التصميمي ويقلل من التكلفة الأولية للمبنى.
وتعتبر عمليات النقل ضمن كل أنشطة دورة حياة المبنى , وتعد عمليات النقل من أهم عوامل نجاح عمليات إنتاج المواد , ورغم أهمية عمليات النقل إلا أنها تزيد من الطاقة المستهلكة طبقا للمسافة , وكذلك يصدر عنها إنبعاثات كربونية ضارة , والجدول (1) يوضح مقدار الطاقة المستهلكة لنقل مواد الإنشاء بإستخدام وسائل النقل تبعا لنوع الوقود.

جدول رقم 1 يوضح مقدار الطاقة المستهلكة لنقل مواد الإنشاء

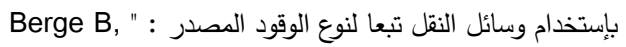
The Ecology of Building Materials",P19, (2009).

\begin{tabular}{|c|c|c|}
\hline الطاقة المستهلكة & طبيعة الوقود & وسيلة النقل \\
\hline $36-33$ & وقود حفري & النقل الجوي \\
\hline $2.2-0.8$ & وقود حفري & النقل البحري \\
\hline $0.9-0.3$ & وقود حفري & النقل البري \\
\hline $0.9-0.6$ & وقود حفري & القطار ات \\
\hline $0.4-0.2$ & كهرباء & \\
\hline
\end{tabular}

ومن الجدير بالذكر أن نقل المنتجات ومواد البناء من أماكن الإنتاج إلي مراكز التوزيع الإقليمية أو تجار مواد البناء تستهلك

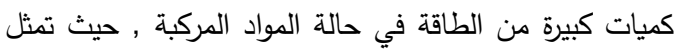

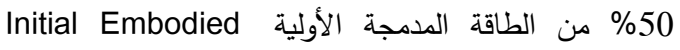
, Energy بينما تقل في حالات الإنتاج المحلي الذي يقلل أيضا الأعباء

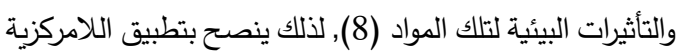

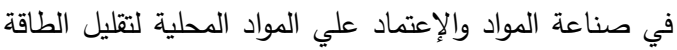
الرمادية المستهلكة كما هو موضح بالثكل رقم (3).

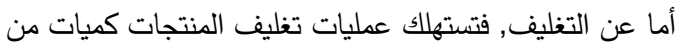

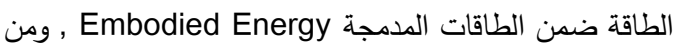
أهم فوائدها الحفاظ علي المنتج وتقليل الهالك أثناء النقل , ولكن من مشكلات التغليف أنه يستهلك كميات كبيرة من المواد في

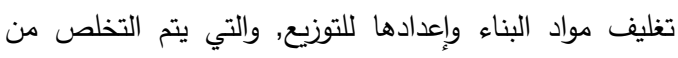

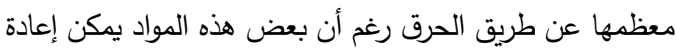

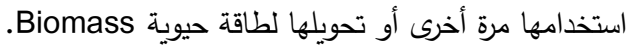


أما إعادة التدوير نثاط يسمح بتدوير مخلفات البناء الصادرة من

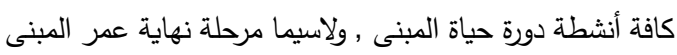

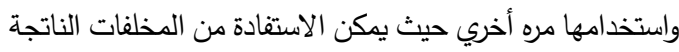

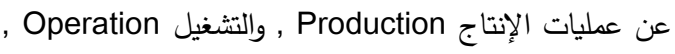

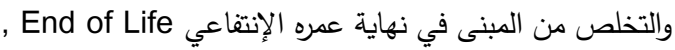
وتدخل في العديد من عمليات الإنتاج كمواد أولية مرة أخري ,

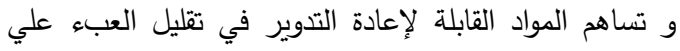
المصادر الأولية لتلك المواد , وتقلل الطاقة المدمجة الأولية Initial Embodied Energy البناء المكونة للمبنى كما هو موضح بالجدول التالي (2) ويصاحب ذلك تقليل إنتاج المخلفات , والملوثات المختلفة للبيئة

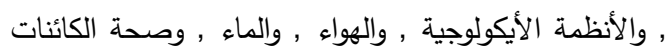

جدول رقم 2 يوضح مقدار ترشيد الطاقة المتهلكة في حالة تدوير مواد الإنثاء لبعض المواد المصدر : Jong-Jin KIM \&Brenda

Rigdon,"Qualities , Use , and Examples of

Sustainable Building Materials", (1998), p 14.

\begin{tabular}{|c|c|c|}
\hline 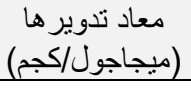 & (ميجاجول/كجم) & المادة \\
\hline 18 & 40 & الحديد \\
\hline 27 & 196 & الألمنيوم \\
\hline 29 & 65 & البوليسترين \\
\hline 56 & 98 & PVC \\
\hline
\end{tabular}

غالأثر البيئي للمئنتج Environmental impact مادة البناء كمنتج ينتج الكثير من الأضرار السلبية علي البيئة

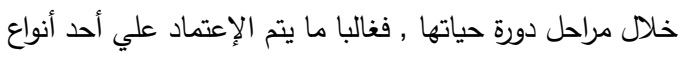

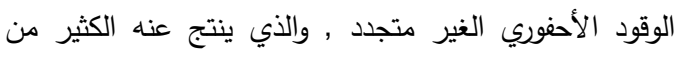

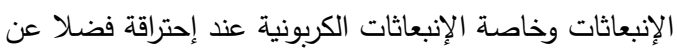

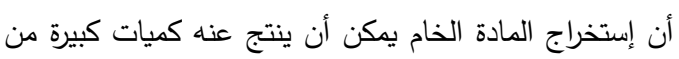

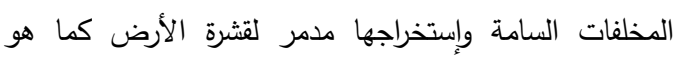
موضح بالجدول رقم (3).
ج- المزايا الخاصة Special Effects تتمثل المزايا الخاصة لأي منتج أو مادة مثلا في (قدرتها في تحمل الحرارة - تحمل الرطوبة - المتانة - بعض الإضافات التهات

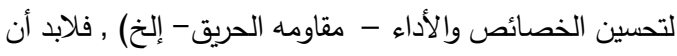

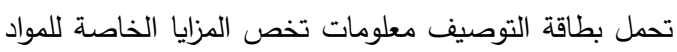

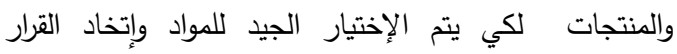
الإقتصادي والبيئي السليم

ح- إمكانية إعادة الإستخدام أو التدوير في نهاية حياة End-of Life (Re-use or recyling) المنتج يجب أن تحمل بطاقة التوصيف معلومات تحدد مدي إمكانية إعادة إستخدام أو تدوير أو تجديد أو تحسين المنتج لتأثير هذه

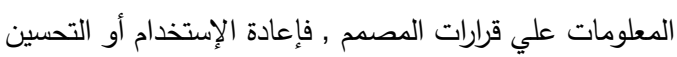

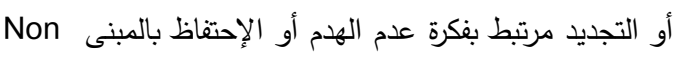
- - Demolition التخلص من المبنى Demolition , فالمقصود بإعادة إستخدام

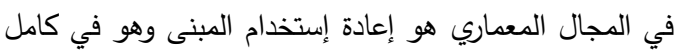

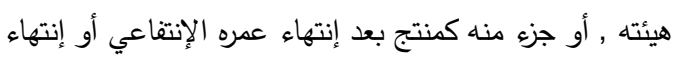

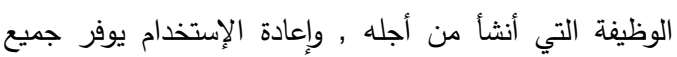

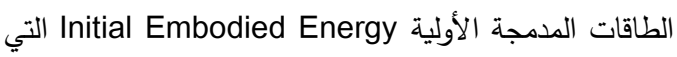
تستهلك في مرحلة الإنتاج Production أحد مراحل دورة حياة المبنى. والثكل البياني التالي (4) يوضح مقدار الطاقة المدمجة

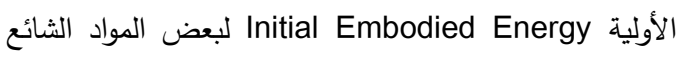
إستخدامها والتي يمكن ترشيدها في حالة إعادة الإستخدام.

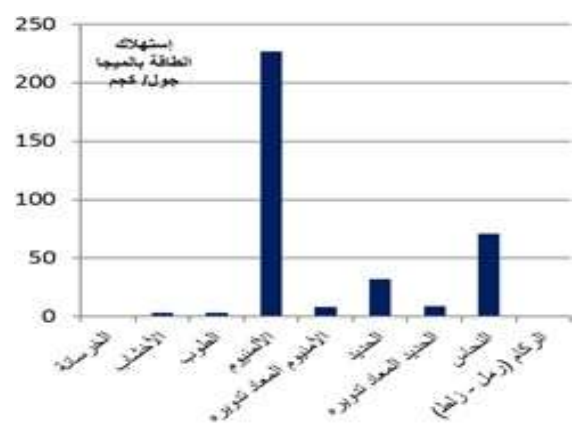

شكل رقم 4 يوضح الطاقة المدمجة الأولية المستهلكة لبعض المواد

الثائع إستخدامها في عمليات الإنثاء المصدر : . Charles J

Kibert, "Sustainable Construction" 2013 p365 
البيئية التقديدية في التهوية والتبريد كالملاقف وخلافه مما له أثرا إقتصاديا سلبيا علي إقتصاديات تشغيل المبنى.

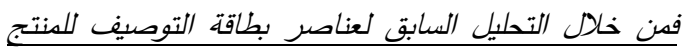

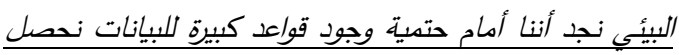

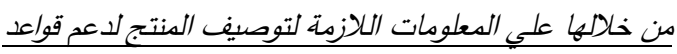
تصنيف المنتجات Product Category Rules (PCRs)

بعد دراسة أليات توصيف المنتج بيئيا EPD وإببلز أهية وجود

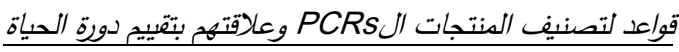

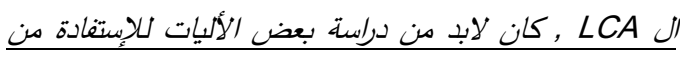
نظام التوصيف البيئي للمنتجات لتحسين أداء المبانسي إقتصاديا,

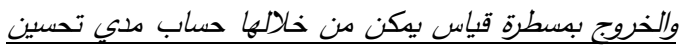

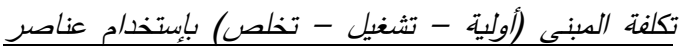
توصيف المنتج البيئي.

أليات الإستفادة من نظام التوصيف البيئي للمنتجات لتحسين

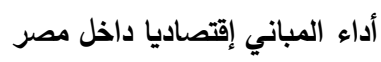

من الدراسة السابقة نجد أن المبنى عبارة عن مجموعة من المواد

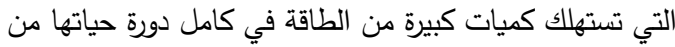

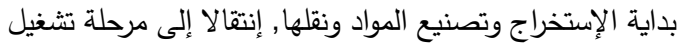

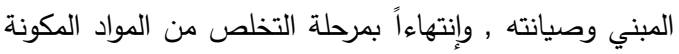

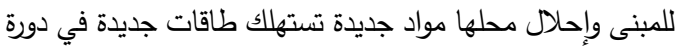
حياة جديدة، فمن المعتاد عند إختيار مواد الإنثاء في مرحلة

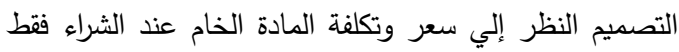

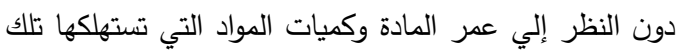
المواد حتى الوصول إلى التشطيب النهائي الصالح للإستخدام وكذلك الأداء الحراري الذي يؤثر على تكاليف تشغيلها بعد ذلك الك الكي

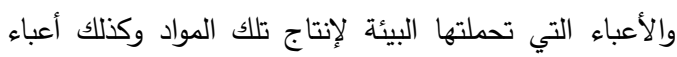
التخلص منها في نهاية عمر المبنى , ودون النظر إلي أمكانية

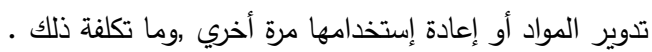

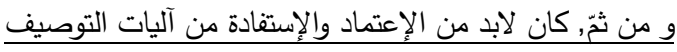
EPD في مساعدة المصمم المعماري في الإختيار الجيد لمواد البناء بثكل يحسن الأداء الإقتصادي والبيئي للمبني علي مدار دروة حياته , وكذلك الإنسـتفادة من قواعد تصــنيف المنتجات
جدول رقم 3 يوضح تأثير مراحل البناء على البيئه. المصدر : Advan

Attmann, O.,Green Architecture Advanced technologies and Materials, McGraw Hill books, United States America, (2010).

\begin{tabular}{|c|c|}
\hline التأثثير على البيئة & النشاط \\
\hline تصدير البيئه النباتيه و الحيوانيه & الأستخر واج \\
\hline استهلاك للطاقه & التصنيع/ التجميع \\
\hline استبعاثات غاز للطاقهى أكسبد الكربون & النقل / التوزيع \\
\hline تلوث ناتج من الأشعاعات أكسيد الكربون & البناء \\
\hline 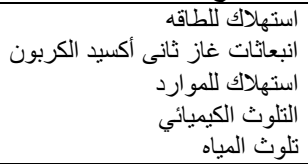 & $\begin{array}{r}\text { Life / الصيانه } \\
\text { cycle building } \\
\text { phase }\end{array}$ \\
\hline التلوث الكيميائي & الهدم \\
\hline انلوثاث غاز المياه الجوفيه & اعادة التدوير \\
\hline
\end{tabular}

بينما المواد التي يتم حصادها من مصادر متجددة أو مستدامة

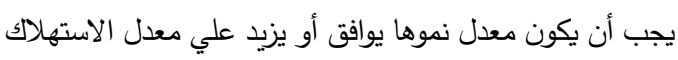

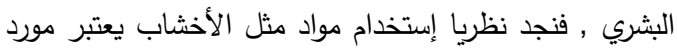

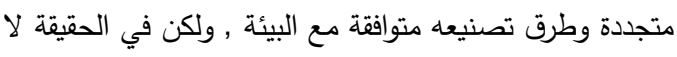
يمكن إعتبار الأخثاب مورد متجدد إلا إذا فاق معدل إنتاجه

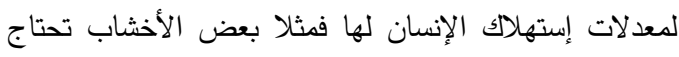

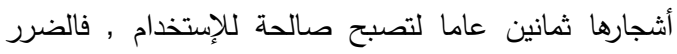
الأيكلوجي الناتج عن إستخدام هذه المواد في البناء يؤدي إلى لى لئي

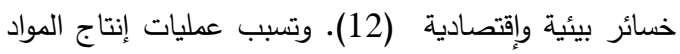

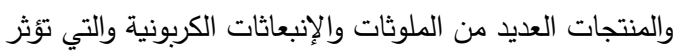

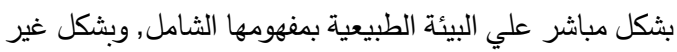

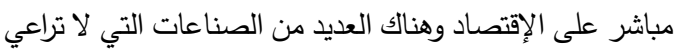
عوامل الأمان وصحة الإنسان, ولازلنا بحاجة للبحث عن أساليب إنتاج أقل خطورة علي البيئة والإقتصاد. فكان لابد من أن تحمل بطاقة التوصيف معلومات تخص الأثر البيئي للمنتج بكل شفافية للحد من إستخدام المواد الملوثة والتي لتئي أفسدت البيئة الخارجية بشكل يحد من الإعتماد علي التطبيقات 
• الحد من تحمض مصادر المياه و الأراضي كأحد الموارد الإقتصادية وعناصر الإنتاج.

• الحد من استنزاف موارد الطاقة غير المتجددة (13)

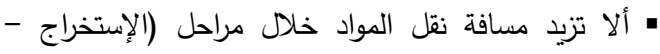

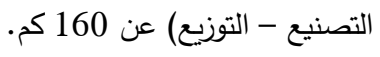

و الهدف الرئيسى من ادراج هذه النقاط الإضافيه لشهادة

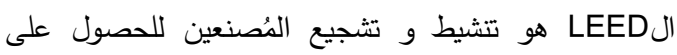

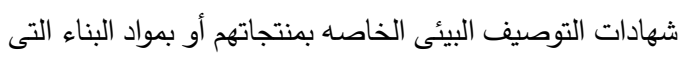

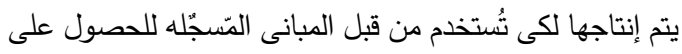
LEED

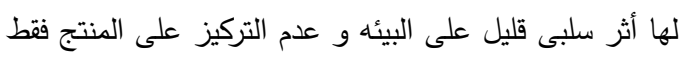
و إنما على تأتيره البيئى و الإقتصادى.

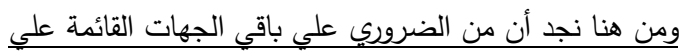
صناعة أليات التيقيم العالمية والمستدامة مثل مركز بحوث البناء لبناء المصري والمعني بتطور الـ GPRS أن تنتهج نفس المنهج التي ولئي

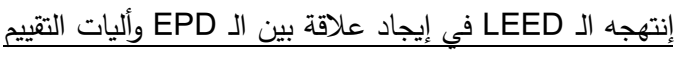
لتشجيع العاملين في قطاع التثيييد علي إستخدام أليات التوصيف

للمنتج بيئيا.

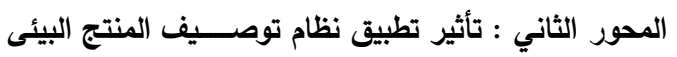
EPD

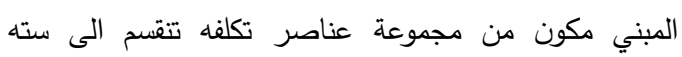

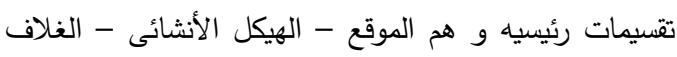

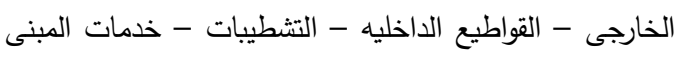
(14) و يندرج تحت كل تقسيم مجموعه من العناصر التى التى لثيه

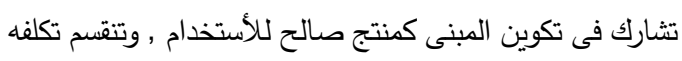

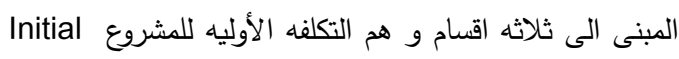
و و تكلفه التشغيل والصيانه Running Cost التخلص Disposal Cost. التكلفه الأوليه للمشروع Initial Cost : تشمل تكلفة مرحلة التصميم وما قبل البناء ومرحلة البناء , وتقليل هذه التكلفه دائماً هدف المالك بهدف الربح المالى دون النظر الى التكلفه البيئيه. تكلفه التشغيل والصيانه Running Cost : هى تقييم الآداء الأقتصادى للمبنى او جزء منه من خلال مجموع التكاليف الثابته

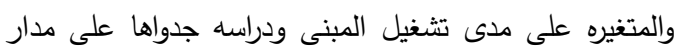

PCRs والمســدامة , لذلك تأتي دراســــة أليات الإنـــتفادة من نظام التوصيف البيئي للمنتجات لتحسين أداء المباني إقتصاديا علي محورين : المحور الأول: علاقه نظام توصيف المنتج البيئى EPD بنظام

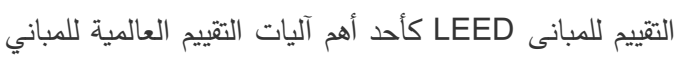

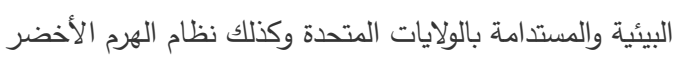

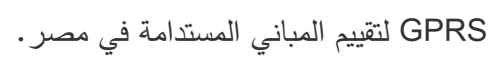

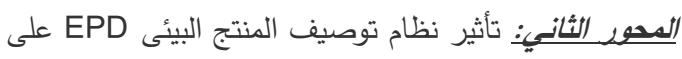

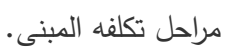

EPD المحور الأول : علاقه نظام توصــيف المنتج البيئى بنظام التقييم للمبانى LEED و الـ الـ

أضاف نظام الـ LEED مجموعة من نقاط التقييم حال إختيار

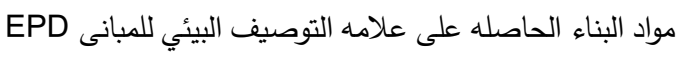

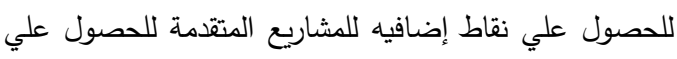
تقييم الـ LEED. حيث أنه تم اضافه نقاط إضافيه (عدد 2 نقطه كحد أقصى) ض ضمن عنصر تقييم المواد و و "Materials and Resources credits in LEED" الموارد لتشجيع مصممي المبنى لتحديد المنتجات من الشركات المصنعة

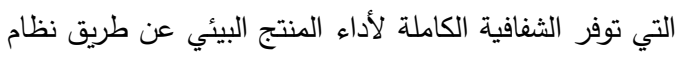
التوصيف البيئى EPD. و يمكن الحصول على النقاط

$$
\text { الإضافية عن طريق : الإنيف }
$$

أولا: للحصول على النقطه الأولي: يجب استخدام ما لا يقل

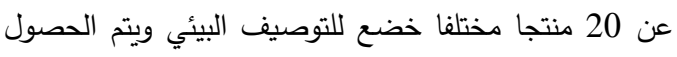
عليها من خمسة مصنعين مختلفين يستوفون معايير التوصيف بألية الـ EPD. ثانيا: للحصول عل النقطة الثانية: يكون من خلال إقرار الحد من التأثير البيئي طبقا لتقييم طرف ثالث محايد بتحقيق المعايير

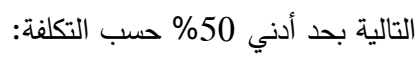
• تقليل انبعاثات الغازات الدفيئة GHG وتقيليل إحتمالية حدوث إحتباس حرارى. • تقليل الأضرار علي طبقة الأوزون Ozone layer 


\begin{tabular}{|c|c|c|c|c|}
\hline \multicolumn{5}{|c|}{ Building Phase مرحلة البناء } \\
\hline تكلفة التخلص & تكفلة التشغيل & اللتلفه الأوليه & 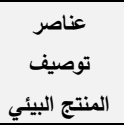 & p \\
\hline 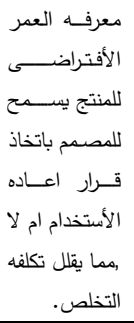 & 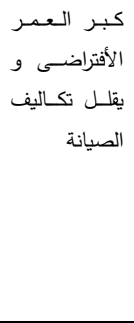 & 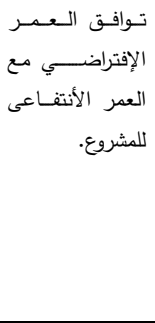 & 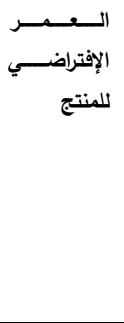 & 1 \\
\hline 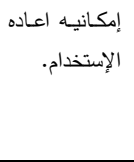 & 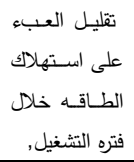 & 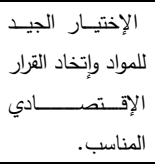 & 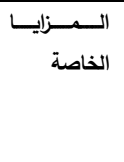 & 2 \\
\hline 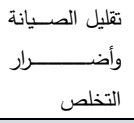 & تقتليل إنبعاثات & مصـــــادر المواد & للمنتج الأثر البيئي & 3 \\
\hline \multicolumn{5}{|c|}{ مرحلة ما بعد البناء Post-Building Phase } \\
\hline تكلفة التخلص & تكفلة التشغيل & التكلفه الأوليه & 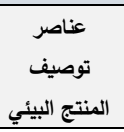 & b \\
\hline 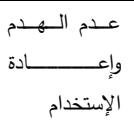 & تقليـلـ أعبــاء & 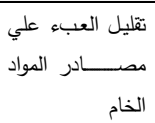 & إلتكانية إعادة الإستخدام أو & 1 \\
\hline 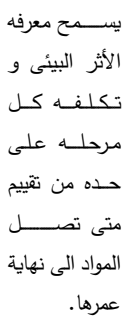 & 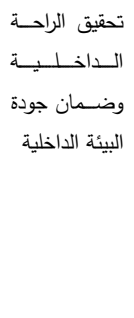 & 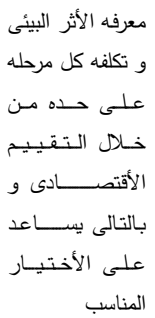 & تلقيـيم دورة & 2 \\
\hline
\end{tabular}

النتائج

- نظراً لزياده الوعى البيئى العالمى و ادرالك مدى تأثير التعدّى

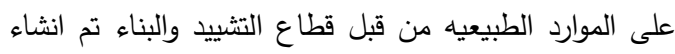

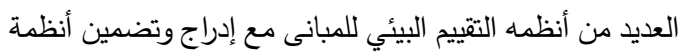

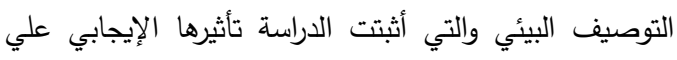
إقتصاديات المبني خلال دورة حياته وعلي مراحل التكلفة
العمر الأنتفاعى للمبنى , ودراسة هذه التكلفه عادة ليست هدف

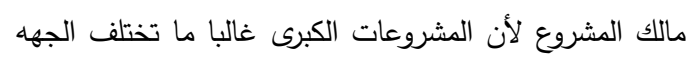
المالكه عن الجها المشغله للمشروع. تكلفه التخلص Disposal Cost : هي التكلفة المرتبطة بالمرحلة التي تتنهي عندها العمر الإفتراضي أو الأنتفاعي

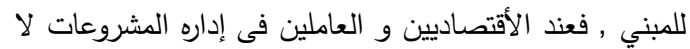

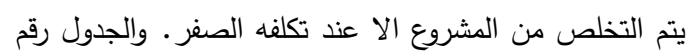
(4) يوضح تأثير استخدام نظام التوصيف البيئى على مراحل التئل

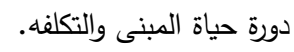
جدول رقم 4 تأثير استخدام نظام التوصيف البيئى على مراحل دورة حياة

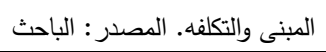

\begin{tabular}{|c|c|c|c|c|}
\hline \multicolumn{5}{|c|}{ مرحلة ما قبل البناء Pre-Building Phase } \\
\hline تكلفة التخلص & تكفلة التشغيل & اللمشلفه الأوليه & توصناص & م \\
\hline 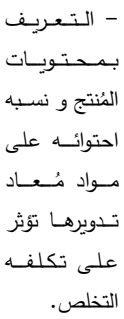 & 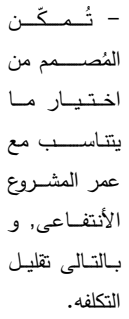 & 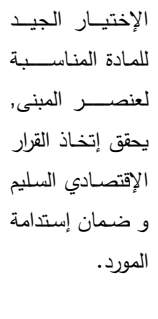 & 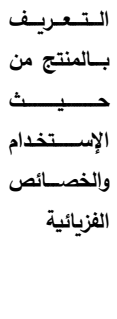 & 1 \\
\hline \# & \# & 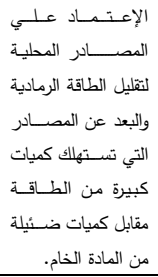 & 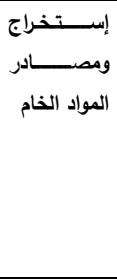 & 2 \\
\hline 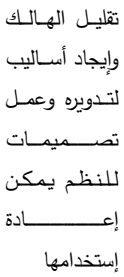 & 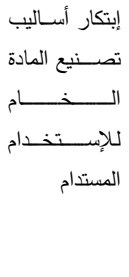 & 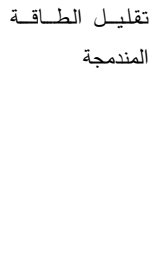 & إنتاج المادة & 3 \\
\hline \# & \# & 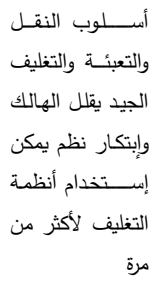 & نقل وتغليف & 4 \\
\hline
\end{tabular}




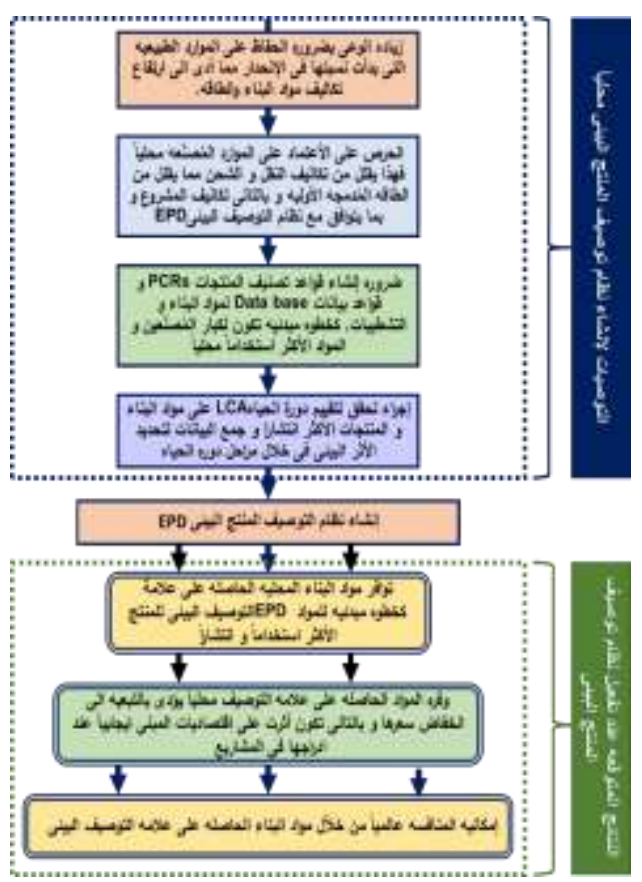

شكل رقم 5 بعض الخطوات المبئيه و التوصيات للأستفادة من تأثير نظام

التوصيف البيئى على إقتصاديات المشروع و السوق المحليه. المصدر:

$$
\text { الباحث. }
$$

- لإتخاذ قرار إدراج مواد البناء الحاصله على علامة التوصيف البيئى فلابد أولا من إدراك المالك والمصمم والمقاول لتأثير هذا

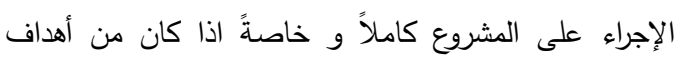

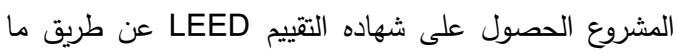
يلى:

أولا: إجراء المزيد من الأبحاث للوصول الى المعلومات الخاصة بخصائص مواد ومنتجات البناء لإنشاء قاعدة بيانات تدعم الحصول على علامه التوصيف البيئى EPD والمساعدة في إنثاء الإعداد لنماذج محاكاه كدراسة مستقبلية لمجموعه من المشروعات المعمارية تختلف في مصادر وطبيعة المواد والأنظمة بشكل مقارني للخروج بنتائج رقمية وأوزان نسبية توضتح مدي تأثير تطبيق مخرجات أنظمة التوصيف البيئي للمواد وتأثيرها علي إقتصاديات المباني خلال دورة حياتها.

ثانيا: دعم رؤية المُصمم لتشمل التكلفة البيئية والإقتصادية لكامل مراحل دوره حياة المنتج وليس الى سعره فقط عند الإختيار
- أثبتت الدراسة أن التوصيف البيئي الجيد للمواد سوف يكون له تأثير واضح علي كل مراحل دورة حياه المبني .. فبالتوصيف البيئي وبمعلومية خصائص المادة يستطيع المصمم الإختيار الجيد لمواد البناء التي لها تأثير بيئي إيجابي ويقلل من تكلفة الإنشاء ويحسن من الأداء أثثاء التشغيل وأثناء التخلص والذي إلي لئي يدعم بدوره تحويل المبني من كونه تقليدي إلي مبني مستدام. - الإعتماد علي أليات التوصيف البيئي لمواد البناء يقلل الطاقة المدمجة والتي تؤثر بشكل مباشر إقتصاديات المبني من خلال التشجيع علي إستخدام وسائل النقل التي تعتمد علي الكهرباء والتشجيع علي إعادة التدوير والإعتماد علي الأساليب اللامركزية في تصنيع وتوزيع مواد البناء. - يختلف التأثير الإقتصادي والتأثير علي تكلفة المباني من من مشروع لأخر حسب مواد البناء المستخدمة ودورة حياة المنتج وإمكانية إعادة الأستخدام. - اتجاه الدول الكبرى فى الصناعه إلى إعتماد أنظمه توصيف المنتج البيئى EPD سيضع الدول المتأخرة عن تطبيق أليات التوصيف أمام عقبات تخص تداول المنتجات خلال التبادل

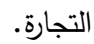
- عدم توافر هذه المواد و المُنتجات يزيد من سعرها لدواعى زياده تكاليف النقل و الثحن و استهلاك الطاقه و هذا يتعارَض مع مفهوم نظام التوصيف البيئى للمنتج EPD القائم على التقليل

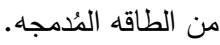
التوصيـات - نظراً لتأثير نظام التوصيف البيئى EPD على اقتصاديات المبنى وعناصر التكلفه المختلفه, ومع ضروره ملاحقه الركب العالمى فى اصدار أنظمه لتضمين البعد البيئى و تقييم دوره الحياة LCA فى مراحل انتاج مواد البناء, وضروره الحد من استتزاف الموارد قدر الإمكان وتقليل الطاقه المدمجه فى عمليات الأنتاج لمواد البناء المختلفه. فلابد من اتخاذ خطوات فى هذا

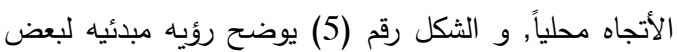
التوصيات و الخطوات الواجب اتباعها لملاحقه الركب العالمى لإن فيما يخُص مواد البناء. 
العمارة والعمران في عقود التحويلات ، جامعة القاهرة

M. Fet et al, "Product category rules and environmental product declarations as tools to promote sustainable products", Volume 11, Issue 2, pp 201-207, June

URL:

https://cdn.scsglobalservices.com/files/pr ogram_documents/SCS_EPD_onesheet.p .df, Accessed June (2020).

URL:

https://www.environdec.com/PCR/Whatare-product-category-rules, Accessed June (2020).

Mary Ann Curran,"Life Cycle

Assessment: Principles And Practice

",Scientific Applications International

Corporation (SAIC), EPA, p.11, (2006).

Business Dictionary Site,"Material

Production":

URL:

https://encyclopedia2.thefreedictionary.co $\mathrm{m} /$ Material+Production\%2C+Sphere+of, Aceessed:July 2, (2020).

Bayer C. et al , " A Guide to Life Cycle

Assessment of Buildings", Georgia Institute of Technology, The American .(Institute of Architects AIA, P47, (2010

Bayer C. et al ,"A Guide to Life Cycle

Assessment of Buildings", by The American Institute of Architects AIA, P.49, ,(2010).

(9)

$$
\begin{aligned}
& \text { ويكون بناءاً على العمر الأنتفاعى للمنتج طبقاً للمواصفات }
\end{aligned}
$$

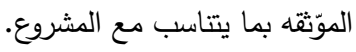

$$
\begin{aligned}
& \text { ثالثا: يجب على المُصمم انتهاج المنهج الثولى و التكاملى فى } \\
& \text { التصميم لدراسة تأثير مواد البناء على عمليه البناء كاملةً. } \\
& \text { رابعا: الإعتماد علي أنظمة التوصيف البيئي للمنتج والتي تساعد } \\
& \text { على إختيار المواد الإنثائيه ومواد التثطيبات بناءاً على التئي } \\
& \text { معلومات موّتقه مما يُّتهل عمليه المُقارنه. }
\end{aligned}
$$

خامسا: يجب إختيار المواد والمنتجات الحاصله على علامة التوصيف البيئى تكون عاليه الجوده الى جانب انها تُحافظ على على على التهات البيئه مما يرفع من القيمه المضافة للمباني إقتصاديا وبيئيا.

سادسا: يجب علي مصنعي مواد البناء الإتجاه نحو إستخدام أليات التوصيف البيئي أثناء صناعة مواد البناء وتوثيق كافة المعلومات عن المواد والمنتجات خلال دورة حياتها لأنها المدخل

الحقيقي لإنجاح وتفعيل أليات تقييم المباني البيئية والمستدامة

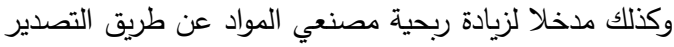
و زيادة تداول المنتج.

T. Ros-Dosdá et al, "Strategic

Environmental Communication Tools", Instituto de Tecnología Cerámica (ITC). Asociación de Investigación de las Industrias Cerámicas (AICE) Universitat .(Jaume I. Castellón. Spain, (2012 Fernandez, "Material Architecture:

Emergent Materials for Innovative Buildings and Ecological Construction", PP. 75, (2006).

علي رأفت " موسوعة الإبداع المعماري : الإبداع

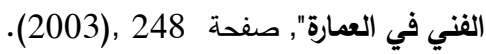
إيهاب محمود عقبة ، "مداخل التصميم البيئي نحو فئه 
ault/files/Guidelines_for_Life_Cycle_Cost

_Analysis.pdf, Accessed May (2020).

Robert P. Charette, Harold E. Marshall, " (14)

UNIFORMAT 2 Elemental Classification

for Building Specifications, Cost

Estimating and Cost Analysis",(1999).
KIM, J. and Rigdon, B. ,"Qualities , Use (12)

, and Examples of Sustainable Building

Materials", p 9. , (1998).

Stanford University Team,"GUIDELINES

FOR LIFE CYCLE COST ANALYSIS"

2005, p.3 URL:

https://sustainable.stanford.edu/sites/def 


\title{
THE IMPACT OF THE ENVIRONMENTAL PRODUCT DECLARATION
} (EPD) SYSTEMS ON THE BUILDINGS' ECONOMIC PERFORMANCE

\author{
Amr Soliman ElGohary
}

Lecturer at Modern Academy for Engineering and Technology-Cairo - Egypt.

\section{Shereen Omar Khashaba}

Lecturer at Canadian International College CIC - Cairo - Egypt.

\begin{abstract}
Building materials have an impact on the development of the architecture and the construction process. As the energy production prices increased through the last few years due to its unavailability, overconsumption, and low percentage of renewables applied in the energy grids; the building initial cost increased as well, due to the increase of the embodied energy of the building materials, Which led the desdigners and investors to abandon sustainable applications in building construction.

As a result, the global trends recommend increasing the added value of buildings, by applying sustainable or environmentally friendly products, and establishing guidelines that drive the inclusion of the economic and environmental aspects in the buildings assessment. The developing countries have to take benefits of that global trend to overcome the economic challenges in the construction sector; thus the research develops a study to the possibility of applying the Environmental Product Declaration (EPD) systems to improve the economics of buildings.

Environmental Product Declaration (EPD) is a standardized way of quantifying the environmental impact of a product or system, and energy use and efficiency; it acts as an identification process to the material that allows decision making process to be based on economic and environmental information, to reduce the burden on resources, and reduce the initial, operational and disposal costs. As well as starting a database that has a clear description of the Egyptian materials commonly used in the manufacture of various building elements.
\end{abstract}

